\title{
Perspectives in the Prevention and Control of Non- Communicable Diseases (NCD)
}

Non-communicable diseases are defined as a set of chronic diseases of major public health importance, such as cardiovascular diseases, cancer, diabetes mellitus, lung diseases such as asthma and chronic obstructive pulmonary disease, the development of which is influenced by one or more common risk factors (e.g., smoking, diets rich in energy and fat, physical inactivity and stress $)^{1}$. The literary meaning that diseases which are not communicable (infectious) are non-communicable diseases is also true. But, the diseases of major public health importance, which are considered eminently preventable and/or controllable, are the diseases included under the term NCDs.

The world is clearly witnessing a growing man-made epidemic of NCDs which is being greatly aggravated by a rapidly ageing global population. It has been estimated that by the year 2020 up to three-quarters of all deaths in the world will result from NCDs, and Ischaemic Heart Disease and Depression will top the list of major public health problems in the world, and that there will be a significant increase in the disease burden from lung cancer ${ }^{2}$. In 2002, non communicable chronic diseases were the leading cause of DALYs in all regions of the world except for Sub Saharan Africa. NCDs accounted for $47 \%$ DALYs and 53\% worldwide deaths in 2002 and the prevalence of NCDs is projected to increase considerably over the next 20 years $^{2}$.

There is considerable diversity in the distribution of NCDs both between countries and regions of the world as well as differences within countries. The rise of NCDs in developing countries is inextricably linked to economic and cultural globalisation. Much of the projected rise in NCDs is preventable, particularly that due to smoking, poor diet, physical inactivity and obesity. Health systems in developing countries face both a growing need for prevention programmes and increasing numbers of individuals requiring treatment ${ }^{3}$.
The conceptual basis for building a framework for prevention and control of non-communicable diseases can be discussed under the following headings ${ }^{2}$ -

Health Determinant and Public Health Perspective The health experience of an individual or a community is influenced by a variety of factors and conditions. The factors which have been found to have the most significant influence on health -for better or worse - are known as 'the determinants of health'. Broadly speaking, these health determinants cover people's genetic predisposition, lifestyles and other behavioural factors, social relationships with families, friends and community, and the powerful forces of the general socio-economic and cultural environment where they learn, play, work and live. These different determinants operate together to influence health and disease status at both the population and individual levels ${ }^{3}$. For public health policy to be optimal, it is important that we have a good understanding of the underlying factors that determine health.

A public heath approach which focuses on population and risk factors rather than on individuals' symptoms or diseases is important to achieve the goal of promoting health and preventing diseases, addressing the underlying factors that determine health, and increasing the effectiveness and efficiency of healthcare system ${ }^{4}$.

Clustering of Risk Factors ${ }^{4-6}$

NCDs are attributed to the complex web of factors described above. Many of these diseases share common behavioural risk factors. For example, four of the most important NCDs are- diseases of the circulatory system, cancer, chronic respiratory diseases, and diabetes mellitus -share three major behavioural risk factors, namely smoking, physical 
inactivity and unhealthy diet, which are mediated through common biomedical risk factors, notably excess weight, hypertension and adverse lipid profile. Preventive actions addressing these common behavioural risk factors will improve the community's health profile, which includes optimal body weight, blood pressure and lipid profile. The community will then be benefited from lower incidence of diseases and better health condition.

\section{Life-course Approach}

Individuals are influenced by factors acting at all stages of the life span and the risk of developing NCD accumulates with age. Life-course approach acknowledges such interactive and cumulative impact of social and biological influences throughout life, particularly the importance of early life factors predisposing to NCD in later years ${ }^{7}$.

Utilising opportunities at each stages of life, it may be possible to have fewer disabilities and reduce premature deaths. The functional capacity, such as muscular strength and cardiovascular output, accumulates in childhood and peaks in adulthood, and then declines in older age. As the rate of functional capacity decline is largely determined by behavioural factors, adopting a healthy lifestyle will help maintain or prevent early decline in functional capacity during older age. For example, stop smoking at age 60, 50, 40 or 30 gains about 3, 6, 9 and 10 years of life expectancy respectively ${ }^{8}$. Thus, it is important to secure growth and development in early life, maintain the highest possible level of function in adult life as well as maintain independence and prevent disability in older life ${ }^{9}$.

\section{Preventive Strategy}

The planning of NCD prevention and health promotion programmes is based on the three levels of prevention: primary, secondary, and tertiary ${ }^{10-11}$.

Primary Prevention: Primary prevention is concerned with measures that prevent the onset of disease. Some of the important strategies under this category include health education, immunisation, environmental measures and social policy. The ultimate goal is to bring about a change in behaviour or factors affecting individuals so that diseases will be prevented from developing. This approach has contributed to some notable examples of successful intervention in public health.

Secondary Prevention: Secondary prevention refers to stopping the progression of a disease after its occurrence, by early detection and diagnosis followed by prompt and effective treatment. The prevention of relapse or recurrence of disease conditions through intervention or attention to lifestyle improvement measures, e.g. smokers to quit smoking after a heart attack is also grouped under this category. Screening, which is one form of secondary prevention, has been more accepted by the general public as a means to "prevent" diseases in recent years ${ }^{12}$.

Tertiary Prevention: Tertiary prevention refers to the rehabilitation of patients with an established disease to minimise residual disabilities and complications and maximise potential years of enjoyable life, thereby improving the quality of life even if the disease itself cannot be cured ${ }^{12}$.

\section{Population-wide versus individual-based Approach}

The distribution of health determinants and risks in a population has implications for successful prevention strategies. While a population-wide strategy for prevention targets at controlling the determinants of health in the population as a whole, an individualbased (also known as high-risk) strategy for prevention identifies high-risk susceptible individuals and offer them some individual protection ${ }^{13}$.

The two approaches have their inherent pros and cons ${ }^{14}$. The population-wide approach seeks to promote healthy behaviour to achieve an overall lowering of the risk in the entire population. The potential gains are comparatively extensive but the effect on each participating individual may not be very significant. In contrast, the individual-based approach may appear more appropriate to the individuals. However, it only has a limited effect at a population level and it does not alter the underlying causes of illness. Such an approach also requires continuous and expensive screening processes to identify the high-risk individuals.

Cardiac rehabilitation programme, which is an example of individual-based approach for prevention, is known to be effective in reducing cardiac deaths. Patients are encouraged to exercise and change their 
lifestyles after having a heart attack or other heart problems and they can be benefited from tailored lifestyle programmes. A systematic review reported that total cardiac mortality was reduced by $26 \%$ to $31 \%$ in the exercise only and comprehensive cardiac rehabilitation groups ${ }^{15}$. Another study showed that lifestyle intervention using such approach reduced the risk of people with impaired glucose tolerance in developing diabetes mellitus by $58 \%$ over 6 years ${ }^{16}$.

With regard to effective interventions using population-wide approach, raising the duties on tobacco products has resulted in a large improvement in population health because fewer people smoke as the price of tobacco rises. Reducing the salt content of processed foods available for sale in the markets, either through legislation or self-regulation of the industry, has resulted in a corresponding reduction in age-specific and sex-specific mean systolic blood pressure ${ }^{17}$. When NCD are prevalent in the community, even modest changes in risk factor levels through population-wide approach will yield significant public health benefit ${ }^{18}$.

There are many examples worldwide on successful mix of population-wide approach and individualbased approach for preventing and controlling NCD. Communities can make major gains once becoming involved in reducing health risk behaviours associated with many chronic diseases. Some of the most notable cardiovascular diseases prevention trials are the Stanford Three-Community Project, North Karelia Project, Stanford Five-City Project, Minnesota Heart Health Program and the Pawtucket Heart Health Program ${ }^{16-18}$. These projects have made known that cardiovascular diseases are preventable through modifications of established risk factors including cigarette smoking, elevated blood lipids, elevated blood pressure and sedentary lifestyle.

The basic premise for this work is that communitywide strategies lead to a reduction in disease rates through changes in individual and community risk factors. Each provides valuable models, diversified methodologies addressing awareness and education, skill-building and advocacy, and strategies for planning and implementing community-based/led programmes. These programmes are cost-effective, easily transferable and have dramatic impacts on health policy development ${ }^{18}$.

\section{Health Disparity}

Disparity in health usually refers to a broad range of differences in health status between population subgroups. Although some disparities in health are inevitable because of genetic and biological make-up in individuals, health disparities are often attributed to differences in personal lifestyle, exposure to material resources and opportunity of receiving healthcare services $^{19}$. For example in China, as the result of increasing affluence and the adoption of western diet, people living in the cities had a 2.7 -fold increase risk of having diabetes mellitus than those living in poor rural area $^{20}$. Striving to minimise the health gap between population subgroups has become a challenge in public health ${ }^{2}$. Thus, an important public health task is to identify the underlying health determinants attributable to health disparities and develop responsive policies for their reduction.

\section{Health Literacy and Social Marketing}

Health literacy is the ability to read, understand, and act on healthcare information. Study has indicated that poor health status is disproportionately high among people with low health literacy ${ }^{21}$. For enhancing the population health, therefore, the health literacy of the whole population needs to be increased. Social marketing, as an effective health promotion method, can motivate people to use health information and change behaviour in ways that promote and maintain good health. Over years, many places including Hong Kong have used social marketing campaigns for health promotion ${ }^{22-23}$.

\section{Setting Health Priority}

Bangladesh has one of the most vulnerable economies, characterized by extremely high population density, low resource base, and high incidence of natural disasters. These have adverse implications for long-term savings, investment, and growth. There is never as much funding as is needed to address all important health problems, so priorities need to be set. Priority setting is imperative for the rational utilisation of resources for public health programmes in a community ${ }^{24}$. However, identification of priority health areas is not easy. Whether or not a particular disease or health condition should be focused and targeted for preventive activities depends on a number of factors.

Over the past two decades, some developed countries have gone through the process of identifying health 
priorities and started working on the identified health priority areas. For example, Australia has selected seven National Health Priority Areas for action, including asthma, cardiovascular health, cancer control, injuries prevention and control, diabetes mellitus, mental health and arthritis and musculoskeletal conditions, while the United States (US) also views heart disease and stroke, cancer and diabetes mellitus the most important health problems ${ }^{25}$. We need to agree what priorities should be set in Bangladesh and what targets need to be met. Before selecting the priorities of NCDs for intervention we have to obtain the population based data through surveillance ${ }^{26}$.

\section{Md Ridwanur Rahman', Emran Bin Yunus², M A Faiz $^{3}$}

${ }^{1}$ Professor of Medicine, BKZ Medical College, Dhaka.ridwanurr@yahoo.com, to be corresponded

${ }^{2}$ Professor of Nephrology, Chittagong Medical College, Chittagong

${ }^{3}$ Professor of Medicine, Sir Salimullah Medical College, Dhaka

(J Bangladesh Coll Phys Surg 2009; 27: 59-62)

\section{References:}

1. Truelsen T, Bonita R. Surveillance of stroke: The WHO STEPwise approach. Summary. Geneva, World Health Organization, 2002.

2. Murray CJL, Lopez AD, The Global Burden of Disease: A Comprehensive Assessment of Mortality and Disability from Diseases, Injuries and Risk Factors in 1990 and Projected to 2020. Harvard University Press. Boston 1996

3. World Bank. Public Policy and the Challenge of Non Communicable Diseases. June 27, 2007. Washington, DC.

4. Braine, Theresa. Mexican billionaire invests millions in Latin American health. Bulletin of the World Health Organization. Volume 85, Number 8, August 2007. Geneva, Switzerland.

5. WHO. Eleventh General Programme of Work. May 2006 Geneva, Switzerland.

6. Preventing chronic diseases: a vital investment - WHO global report. Geneva: World Health Organization, 2005.

7. Anderson, GF, Chu, E. Expanding Priorities - Confronting Chronic Disease in Countries with Low Income. New England Journal of Medicine. January 2007. 357: 209-211.

8. Anderson, Gerard F., Hyder, Adnan A. Non Communicable Disease and Injuries in Eastern Europe and Eurasia. USAID. October 2006.
9. Disease control priorities in developing countries. 2nd ed Washington, DC: World Bank, 2006.

10. Anselmi, M, Avanzini, F et. Al. Treatment and control of arterial hypertension in a rural community in Ecuador. Lancet. April 5; 361 (9364): 1186-7.

11. Gagliardino, JJ, Etchegoyen, G et. Al. A Model Educational Program for People with Type 2 Diabetes. Diabetes Care. 24: 1001-1007, 2001.

12. Arauz, AG, Sanchez G, Padilla G. A community diabetes educational intervention at the primary care level. Revista Panamericana Salud Publica.

13. Teutsch, S. M. and R. E. Churchill, eds. 2000. Principles and Practice of Public Health Surveillance, 2nd edition. New York: OUP.

14. Halperin, W., E. L. Baker, P. R. Monson, eds. 1992. Public Health Surveillance. New York:

15. http://www.who.int/csr - WHO's Communicable Disease Surveillance website

16. http://www.who.int/csr/resources/publications/ csrpublications /en/index 19.html for key WHO documents on surveillance

17. World Health Organization. The World Health Report: 2002: Reducing risks, promoting healthy life. 2002. World Health Organization.

18. Tunstall-Pedoe $\mathrm{H}$ for the WHO MONICA Project. World Health Organization MONICA Project (Monitoring Trends and Determinants in Cardiovascular Disease): a major international collaboration. J Clin Epidemiol 41, 105-114. 1988.

19. World Health Organization. Global strategyfor the prevention and control of noncommunicable diseases. Report by the Director General. A53/4. Fifty-third World Health Assembly, May 2000. World Health Organization. 2000. Geneva.

20. Eastern Stroke and Coronary heart Disease Collaborative Group. Blood pressure, cholesterol, and stroke in eastern Asia. Lancet 352, 1801-1807. 1998.

21. Thacker SB and Berkelman RL. Public health surveillance in the United States. Epidemiol Rev 10, 164-190. 1988.

22. McQueen DV. A world behaving badly: the global challenge for behavioral surveillance. Am.J.Public Health 1999;89:1312-14.

23. Bonita R, Strong K, de Court. From surveys to surveillance. Rev Panam.Salud Publica 2001;10:223-25.

24. Truelsen, T., Bonita, R., and Jamrozik, K. - Surveillance of stroke: a global perspective. - Int J Epidemiol 2001 Oct; 30 Suppl 1:S11-6. S11-S16. 10-1-0001.

25. World Health Report 2005: World Health Organization. Geneva 2005.

26. Faiz M A (2008). Surveillance and prevention of noncommunicable diseases (NCDs) in Bangladesh (Editorial). J Medicine 9:1-2 